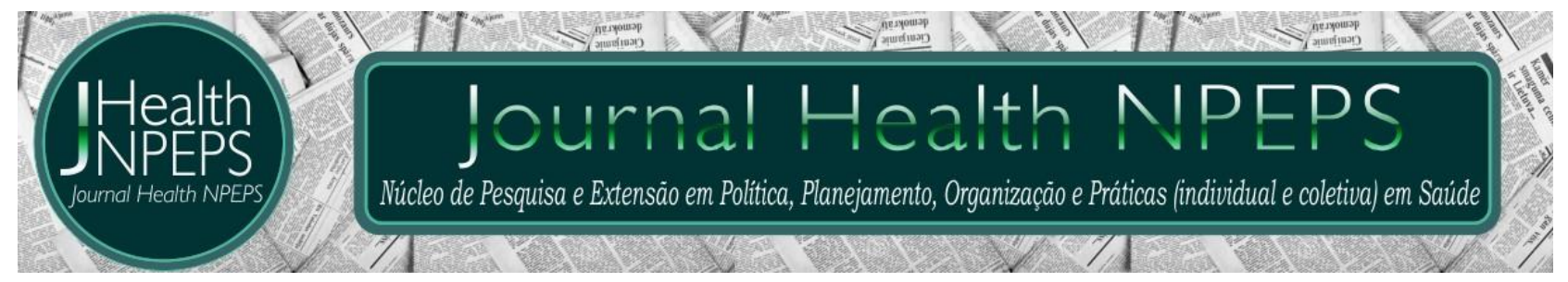

http://dx.doi.org/10.30681/252610103016

ARTIGO DE REVISÃO

\title{
Conceitos, manifestações clínicas e cuidados imediatos na disreflexia autonômica
}

Concepts, clinical manifestations, and immediate care in autonomical disreflexia

\begin{abstract}
Conceptos, manifestaciones clínicas y cuidados inmediatos en la disreflexia autonómica
\end{abstract}

\section{Luene de Albuquerque Vasconcelos ${ }^{1}$, Letícia Kelly Araújo de Souza², Rafaelly Ferreira de Moura ${ }^{3}$, Luiz Paulo Vicente Freire ${ }^{4}$, Luciana Maria de Morais Martins Soares ${ }^{5}$}

\begin{abstract}
RESUMO
Objetivo: revisar a literatura a respeito da disreflexia autonômica e apresentar conceitos, manifestações clínicas e cuidados imediatos diante dessa síndrome. Método: revisão integrativa da literatura realizada no período de maio a junho de 2018. Para a busca de dados foram utilizadas as bases de dados SciELO, PubMed e Medline. Resultados: foram selecionados quinze artigos publicados no período de 2013-2018, em português, espanhol e inglês, que contemplaram em seus títulos e/ou resumos/conteúdo o termo disreflexia autonômica e/ou reabilitação e/ou traumatismo da medula espinal e/ou seus respectivos unitermos, disponibilizados na íntegra. Quanto ao ano de publicação, observou-se que 33,33\% dos artigos selecionados foram publicados em 2017; em relação ao idioma de publicação, a língua inglesa predomina em $73,33 \%$ deles; no que tange ao canal de publicação, a revista The Journal of Spinal Cord Medicine apresenta $20 \%$ dos artigos escolhidos. Quanto às abordagens dos estudos, verificou-se a determinação de conceitos, manifestações clínicas e cuidados imediatos como temáticas mais apresentadas. Conclusão: foi possível observar que a disreflexia autonômica é uma síndrome de grandes limitações, fatores etiológicos e necessidades de cuidados especializados. Vale mencionar que há uma grande escassez de estudos sobre o tema.
\end{abstract}

\footnotetext{
${ }^{1}$ Estudante do Curso de Fisioterapia da UNINASSAU, Departamento de Fisioterapia. João Pessoa, Paraíba, Brasil. Email luene a.v@hotmail.com ORCID ID: https://orcid.org/0000-0003-3830-3739 Autor principal - Endereço para correspondência: Rua Ana Nery, № 77, Tambay- Bayeux- Paraíba, Brasil. CEP: 58307-560.

${ }^{2}$ Estudande do curso de Fisioterapia da UNINASSAU, Departamento de Fisioterapia. João Pessoa, Paraíba, Brasil. Email leticia araujo.souza@hotmail.com ORCID ID: https://orcid.org/0000-0002-6767-8209

${ }^{3}$ Estudande do curso de Fisioterapia da UNINASSAU, Departamento de Fisioterapia. João Pessoa, Paraíba, Brasil. Email rafaelly.jampa@hotmail.com ORCID ID: https://orcid.org/0000-0003-0106-2345

${ }^{4}$ Estudande do curso de Fisioterapia da UNINASSAU, Departamento de Fisioterapia. João Pessoa, Paraíba, Brasil. Email luizpaulo brito@hotmail.com ORCID ID: https://orcid.org/0000-0002-1436-6228

${ }^{5}$ Fisioterapeuta. Doutora em Evolução Humana: antropologia física e forense. Docente do curso de Fisioterapia da UNINASSAU - João Pessoa e Centro Universitário de João Pessoa (UNIPE). João Pessoa, Paraíba, Brasil. E-mail luciana_momaso@hotmail.com ORCID ID: https://orcid.org/0000-0002-6559-9558
}

Este artigo está licenciado sob forma de uma licença Creative Commons Atribuição 4.0 Internacional, que permite uso irrestrito, distribuição e reprodução em qualquer meio, desde que a publicação original seja corretamente citada 
Descritores: Disreflexia Autonômica; Reabilitação; Traumatismos da Medula Espinal.

\begin{abstract}
Objective: to review the literature on autonomic dysreflexia and to present concepts, clinical manifestations and immediate care before this syndrome. Method: it is an integrative literature review, performed in the period from may to june 2018. Data search was performed using the SciELO, PubMed and Medline databases. Results: fifteen articles published in the period of 2013-2018 in Portuguese, Spanish and English were selected, which included in their titles and/or abstracts/contents the term autonomic dysreflexia and/or rehabilitation and/or spinal cord injuries and/or their respective uniterms, available in full. As for the year of publication, it was observed that $33.33 \%$ of the articles selected were published in 2017; in relation to the language of publication, the English language predominates in $73.33 \%$ of them; with regard to the publication channel, The Journal of Spinal Cord Medicine presents 20\% of the articles chosen. Regarding the approaches of the studies, the determination of concepts, clinical manifestations and immediate care were the most presented themes verified. Conclusion: it was possible to observe that autonomic dysreflexia is a syndrome of major limitations, etiological factors and specialized care needs. It is worth mentioning that there is a great shortage of studies on the subject.

Descriptors: Autonomic Dysreflexia; Rehabilitation; Spinal Cord Injuries.
\end{abstract}

\title{
RESUMEN
}

Objetivo: Revisar la literatura acerca de la disreflexia autonómica y presentar conceptos, manifestaciones clínicas y cuidados inmediatos ante este síndrome. Método: es una revisión integrativa de la literatura, realizada en el período de mayo a junio de 2018. Para la búsqueda de datos se utilizaron las bases de datos SciELO, PubMed y Medline. Resultados: fueron seleccionados quince artículos publicados en el periodo 2013-2018, en portugués, español e inglés, cuyos títulos y/o resúmenes/contenido presentaba el término disreflexia autonómica y/o rehabilitación y/o traumatismo de la médula espinal y/o sus respectivos unitermos, disponibles en su totalidad. Se verificó que con respecto al año de publicación hubo un contingente mayor de publicaciones en el año $2017(33,33 \%)$, en lengua inglesa $(73,33 \%)$, mientras que la revista donde la temática fue más abordada fue The Journal of Spinal Cord Medicine (20\%). En cuanto a los enfoques de los estudios, se verificó la determinación de conceptos, manifestaciones clínicas y cuidados inmediatos como temáticas más presentadas. Conclusión: fue posible observar que la disreflexia autonómica es un síndrome de grandes limitaciones, factores etiológicos y necesidades de cuidados especializados. Vale mencionar que hay una gran escasez de estudios sobre el tema.

Descriptores: Disreflexia Autonómica; Rehabilitación; Traumatismos de la Médula Espinal.

\section{INTRODUÇÃO}

A lesão medular (LM) é uma categoria de disfunção neurológica complexa que envolve várias sintomatologias clínicas incapacitantes, em decorrência de agressões à medula espinal, que comprometem sua anatomia e função ${ }^{1}$. Esse acometimento pode vir a gerar ausência ou diminuição da sensibilidade e da força muscular, podendo levar a Journal Health NPEPS. 2018 jul-dez; 3(2):618-633. 
distúrbios neurovegetativos das partes do corpo que se encontram abaixo da lesão e ainda comprometendo os sistemas urinário, intestinal, respiratório, circulatório, sexual e reprodutivo².

A LM pode ocorrer de forma traumática e não traumática, no qual a traumática se caracteriza por haver um inter-rompimento das vias ascendentes e descendentes que carregam as informações entre o cérebro e a coluna vertebral decorrente de algum fator externo ${ }^{3}$. As lesões traumáticas correspondem aproximadamente $80 \%$ dos casos e podem ser causadas por acidentes automobilísticos ou veículos a motor, quedas de altura e mergulho em água rasas e, sobretudo ferimentos por arma de fogo 4 .

As causas não traumáticas (internas) da LM, podem ser decorrente de tumores intra e extra medulares; fraturas patológicas causadas por: metástases vertebrais, tuberculose, osteomielite e osteoporose; estenose de canal medular, deformidades graves da coluna; hérnia discal; isquemia; doenças infecciosas e autoimunes perfazendo cerca de $20 \%$ dos casos $^{4}$.

Logo após a LM, todos os neurônios situados abaixo do nível da lesão passam pela fase do choque medular. Esta fase é caracterizada por uma flacidez associada a déficit sensorial, ausência dos reflexos medulares e alterações de termorregulação, sendo assim, classificada como fase aguda da lesão. Depois de cessada a fase aguda, os neurônios se tornam gradativamente mais hiperativos em consequência da ausência de inibição descendente que podem durar apenas dias ou em média de 4 a 12 semanas para a deliberação $0^{5,6}$.

Além disso, a LM pode ser completa ou incompleta. É considerada completa quando há uma cessação da passagem de estímulos nervosos pela medula espinal com desaparecimento de movimento voluntário abaixo do nível da lesão, ocasionando danos sensoriais e motores. A lesão incompleta se caracteriza pela permanência de alguns movimentos e sensibilidades nas partes corporais abaixo da lesão devido à preservação do funcionamento de algumas raízes nervosas ${ }^{7}$.

De acordo com o nível da LM, ela poderá ser classificada em: tetraplegia ou paraplegia, o qual o termo tetraplegia é utilizado para descrever uma lesão dos segmentos cervicais da medula espinhal, que resulta em diferentes graus de perda funcional do pescoço, tronco, membros superiores e inferiores, e, a paraplegia, que se refere a uma lesão nos segmentos do tórax, lombar, sacral, cone medular ou na cauda 
equina, que resulta em perda de diferentes graus de controle dos membros inferiores e tronco sem envolvimento dos membros superiores ${ }^{8}$.

Segundo Roque et $\mathrm{al}^{9}$ a LM ocasiona sérias complicações, não só sensório-motora já mencionadas acima, como também autonômicas, que representam mais chances de agravamento dos déficits neurológicos, motores e sensitivos com graves repercussões funcionais. São consideradas como disfunções autonômicas: choque medular, disritmia cardíaca, hipotensão ortostática, desregulação térmica e disreflexia autonômica (DA).

Diante das alterações autonômicas citadas, a DA se destaca por desencadear outros sinais e sintomas, que geram grande impacto na saúde funcional da pessoa acometida com sequela de LM e nível de lesão acima da sexta vértebra torácica - T69 .

Assim, a DA consiste em uma complexa síndrome que pode se desenvolver entre $20 \%$ a $70 \%$ das pessoas acometidas com LM acima do nível T6 e é difícil que se desenvolva se a lesão estiver abaixo de T10. Como resultado se identifica a desregulação do sistema nervoso autônomo que induz a uma resposta autonômica não coordenada e que pode proceder em um episódio hipertensivo potencialmente lento quando há um estímulo nocivo abaixo do nível da lesão da medula espinal ${ }^{10}$.

Essa síndrome traz sinais e sintomas que incluem aumento súbito da pressão arterial (PA), bradicardia, sudorese intensa, rubor da pele acima do nível da lesão, pele fria e pálida abaixo do nível da lesão. Pode apresentar crises hipertensivas ou hipertensão não controlada, sendo assim apresentando um alto risco de vida ${ }^{11}$. Além disso, a DA é de grande importância clínica, pois pode causar consequências graves, como acidente vascular cerebral, convulsões e morte, se não tratada ${ }^{8}$.

Diante do exposto e considerando a vivência durante a prática clínica em relação às pessoas com LM, principalmente com lesão em nível de torácica alta, podem vir apresentar a DA, na qual se não for tratada de forma adequada, pode inclusive levar ao episódio de morte, se faz a seguinte pergunta norteadora: quais os sinais e sintomas que a DA apresenta e quais os cuidados que devem ser tomados?

A partir disso, o presente trabalho tem por objetivo revisar a literatura a respeito da DA e apresentar conceitos, manifestações clínicas e cuidados imediatos diante dessa síndrome.

\section{MÉTODO}


Foi realizada uma revisão integrativa de literatura, com características qualitativa e descritiva realizada por meio de acesso a publicações científicas, nas bases de dados: SciELO, Pubmed e MEDLINE, utilizando como descritor pela de busca na Biblioteca Virtual em Saúde (BVS) "Disreflexia autonômica" AND "Reabilitação" AND "Traumatismos da Medula Espinal" e seus respectivos unitermos na língua inglesa.

Para a efetivação dessa revisão, foram delimitadas as seguintes fases metodológicas: $1^{\circ}$ fase: Elaboração da pergunta norteadora; $2^{\circ}$ fase: Busca ou amostragem na literatura (seleção dos artigos); $3^{\circ}$ fase: Coleta de dados; $4^{\circ}$ fase: Análise crítica dos estudos incluídos; $5^{\circ}$ fase: Discussão dos resultados e $6^{\circ}$ fase: Apresentação da revisão integrativa. Para não haver discrepâncias, apenas a autora principal, buscou e selecionou os artigos.

Foram considerados fatores de inclusão: artigos publicados no período de 20132018, em português, espanhol ou inglês, que contemplaram em seus títulos e/ou resumos/conteúdo o termo disreflexia autonômica e/ou reabilitação e/ou traumatismo da medula espinal e/ou seus respectivos unitermos e também disponibilizados na íntegra.

Ao final da análise os dados, os mesmos foram organizados em quadros contendo período, ano de publicação título, autores e resultados gerais formando o corpus da revisão integrativa. Além disso, a discussão dos resultados foi estruturada respondendo duas questões norteadoras: 1) De acordo com a literatura, como a Disreflexia Autonômica pode ser conceituada? 2) Quais os fatores desencadeantes e medidas de cuidado diante da Disreflexia Autonômica?

\section{RESULTADOS}

Com relação aos procedimentos metodológicos, foram realizados: a) identificação dos artigos ( $n=412)$ através da leitura dos títulos dos estudos identificados na busca; b) Triagem, na qual foi realizada a leitura dos resumos ( $n=147)$ e c) Seleção e análise, onde os artigos selecionados foram lidos na íntegra $(n=15)$.

Referente à etapa de seleção dos artigos, foram realizadas leituras para a realização de resumos e fichamentos, para aperfeiçoar o entendimento e aprendizagem referente ao presente tema. Quanto aos artigos selecionados, verificou-se que com relação ao ano de publicação (Gráfico 1), verificou-se um contingente maior de 
publicações no ano de 2017 (33,33\%), ao passo que a revista onde a temática foi mais abordada (Gráfico 2) foi The Journal of Spinal Cord Medicine (20\%).

Gráfico 1- Distribuição da amostra quanto ao ano de publicação.

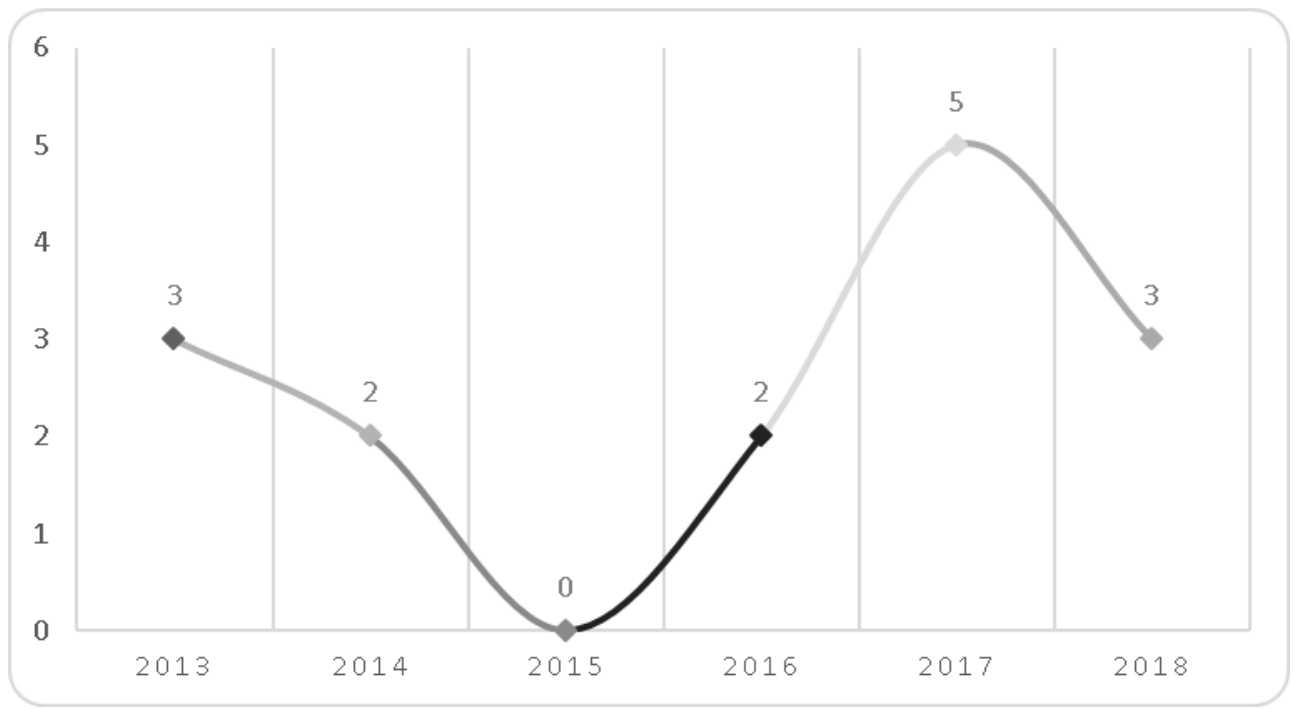

Gráfico 2 - Distribuição da amostra por revista de publicação.

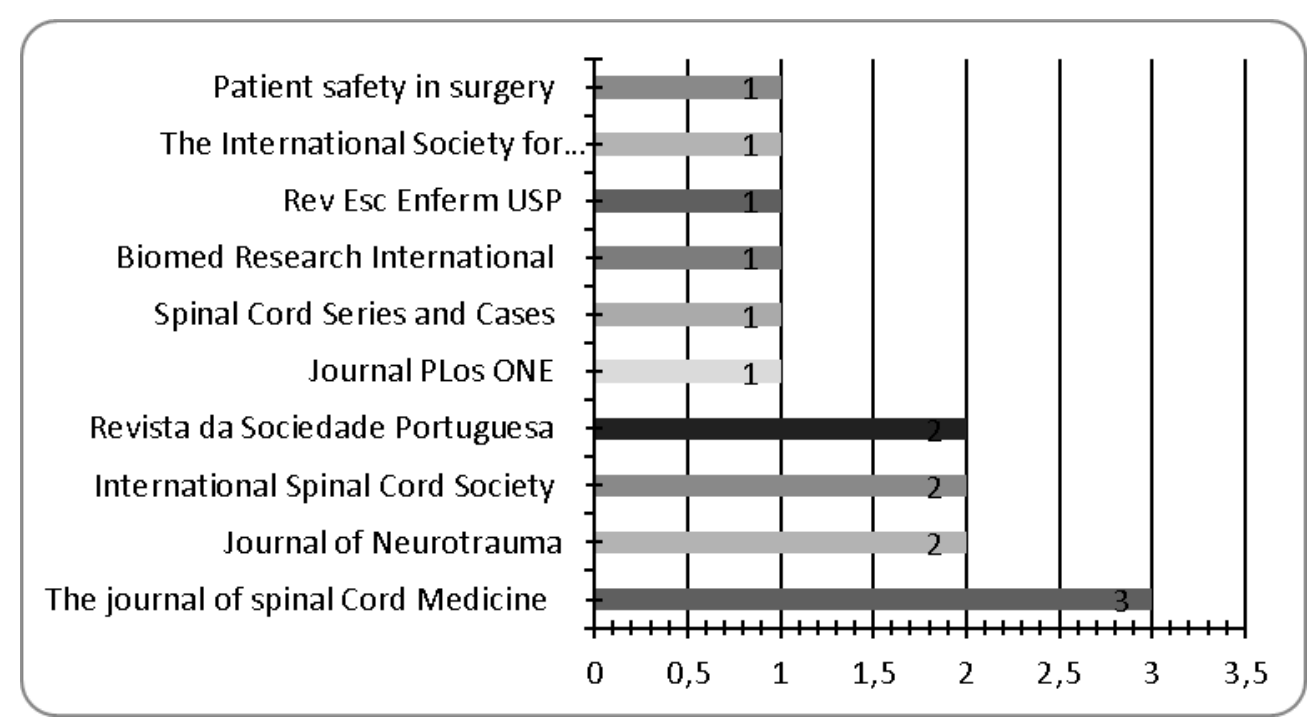

Por fim, as publicações foram analisadas e organizadas em um quadro contendo os seguintes itens: autores, título do artigo, amostra e resultados, formando o corpus da revisão integrativa.

\section{DISCUSSÃO}

\section{Conceituação da Disreflexia Autonômica}


Disreflexia autonômica (DA) é uma desarmonia do sistema nervoso autonômico (SNA), que geralmente acometem indivíduos com níveis elevados de lesão medular (LM). A DA é uma disfunção aguçada com sintomatologia diversificada podendo ser sintomas leves ou graves ${ }^{11,19}$. A DA decorre da cessação do controle supraespinal do sistema nervoso autônomo simpático (SNAS), sendo considerada uma síndrome de acionamento maciço, automático e descontrolado desse sistema ${ }^{9,12}$.

A DA está presente em $70 \%$ dos indivíduos com lesões medulares altas e completas. A incidência e a seriedade são caracterizadas por diversos elementos como nível de lesão, integridade da lesão e o período após o dano ${ }^{13}{ }^{14}$. No entanto, de acordo com Roque et $\mathrm{al}^{9}$, a DA pode se fazer presente em lesões tanto completas quanto incompletas, cujas sintomatologias se apresentam mais marcantes na fase crônica da LM. Assim, é infrequente ocorrer o surgimento dos sintomas no primeiro mês de lesão, porém, a DA pode ocorrer em qualquer momento após o primeiro mês de LM. Inskip et al ${ }^{15}$ menciona que a DA é presente em até $90 \%$ das pessoas com padrões de tetraplegia ou paraplegia alta, no qual ocorre só acima o nível do sistema simpático.

Segundo $\mathrm{Chan}^{8}$, a DA é uma síndrome de emergência, que deve ser tratada para evitar complicações ainda mais graves que a própria DA pode vir a causar, como por exemplos um acidente vascular encefálico (AVE), convulsões e até mesmo a morte. Nesse contexto, Andrade et al ${ }^{16}$ ressaltam que a DA consiste em uma condição de grande importância clínica, no qual é geralmente fatal, podendo ser desencadeada em pacientes com LM.

De acordo com estudo ${ }^{17}$, a DA pode desencadear algumas complicações tais como: infarto agudo do miocárdio (IAM), hemorragia cerebral, convulsões e dentre outros. Vale salientar que nos casos de níveis mais graves da patologia apresenta sinas e sintomas mais rigorosos. Além disso, Lagarto e Pina ${ }^{12}$, relevam que os episódios de DA podem desencadear algumas complicações graves devido ao aumento súbito e sustentados da pressão arterial (PA), presença de hemorragia intracraniana, isquemia do miocárdio, hemorragia retiniana, convulsões e coma.

Conforme publicação recente ${ }^{14}$, a DA pode ser caracterizadas pela apresentação das seguintes manifestações: elevação dos níveis pressóricos cerca de $20-30 \mathrm{mmHg}$, que consiste em umas das principais características dessa desordem, a diaforese (sudorese excessiva), bradicardia, que pode ser precedido de taquicardia e fibrilação atrial. Vale Journal Health NPEPS. 2018 jul-dez; 3(2):618-633. 
mencionar que a gravidade e extensão das sintomatologias irão depender da magnitude dessas manifestações.

Como as crises hipertensivas são bem marcantes, resoluto da elevação de 20 $\mathrm{mmHg}$ na pressão artéria sistólica (PAS) e diastólica basal, é importante realizar o acompanhamento da PA destes pacientes, em particular os tetraplégicos, que tende a ser diminuída (pela vasoplegia). Deste modo, níveis pressóricos considerados normais para a população geral é de $120 \times 80 \mathrm{mmHg}$ ou 130 × $90 \mathrm{mmHg}$ podendo ser medidas elevadas para essa classe de pacientes ${ }^{18}$.

$\mathrm{Na}$ literatura pode-se encontrar discrepância quanto aos valores pressóricos de referência, onde para autores ${ }^{19}$ deve-se considerar aumento de cerca de mais de 20 a 40 $\mathrm{mmHg}$, ao passo que, para outros pesquisadores ${ }^{14}$ e Wang et $\mathrm{al}^{13}$ a elevação dos níveis pressóricos é cerca de 20-30 mmHg acima de base sistólica do paciente. De toda maneira o valor mínimo considerado entre os autores é de $20 \mathrm{mmHg}$. Roque et al ${ }^{9}$ ainda acrescentam categorias da elevação dos níveis pressóricos que variam de leve a grave na qual: na forma leve se observa aumento ou leve da PA é inferior a $40 \mathrm{mmHg}$; moderada é cerca de mais de $40 \mathrm{mmHg}$ e menor que $180 \mathrm{mmHg}$, e, grave mais de $180 \mathrm{mmHg}$.

Estudos $^{9,12}$ acrescentam as cefaleias, diaforese na região da cabeça e pescoço, rubor e congestão nasal. Esses mesmos autores ${ }^{12}$ afirmam que essa desordem pode apresentar também visão turva, vasoconstrição e piloereção (abaixo do nível da LM), diaforese (acima do nível da LM), disritmias, ansiedade e naúseas.

Se esta hipertensão desencadeada pela DA, não for identificada de imediato e se estímulo que está desencadeando não for eliminado rapidamente, pode consequentemente causar nesses indivíduos uma possível AVE, complicando mais seu estado de saúde ${ }^{20}$.

É comum a DA vir associada a hipertensão e ser associada a bradicardia, porém estudos mostram que ainda há controvérsias relacionadas a essa FC. Estudiosos ${ }^{21}$ ressaltam que existem incidências iguais tanto para a bradicardia quanto para a taquicardia.

Outros pesquisadores ${ }^{8,11}$ acrescentam que além das manifestações citadas anteriormente, identificam-se pele fria e pálida abaixo do nível da $L M$, trombose venosa profunda, no qual tem grande probabilidade de induzir a uma embolia pulmonar potencialmente até a morte. 


\section{Fatores desencadeantes e medidas de cuidado diante da Disreflexia Autonômica}

Comumente os fatores etiológicos que podem desencadear a DA são as lesões traumáticas que incluem a LM, e, causas não traumáticas que se referem a neoplasias, patologia de origem vascular como problema arteriovenoso e má formação, patologias degenerativas como a siringomielia, patologias inflamatórias incluindo a mielite transversa e encefalomielite ${ }^{11}$.

Para alguns pesquisadores ${ }^{10}, 85 \%$ dos casos de DA é decorrente a fatores de origem urológica, bexiga distendida ou devido a infecção do trato urinário ou até mesmo um cateter de Foley obstruído. Vaidyanathan et al $^{22}$ acrescentam que a DA pode ser desencadeada por siringomielia, evacuação manual, carga fecal grave de cólon, espondilolistese, coluna de Charcot e lombar neuropática.

Para Roque et $\mathrm{al}^{9}$, os fatores desencadeantes são diversos, no qual os mais comuns são do foro vesical relacionando a distensão vesical, principalmente pelo esvaziamento da bexiga inadequado ou impactação fecal e lesões, como lesões por pressão, patologias abdominais e hemorroidas e dentre outros fatores assim como fraturas, orgasmo e trabalho de parto. Andrade et $\mathrm{al}^{16}$, acrescentam que essa desordem também pode ser decorrente da dismenorréia, onicocriptose e condições musculoesqueléticas indetectáveis.

De acordo com o Ministério da Saúde ${ }^{18}$, Huang et $\mathrm{al}^{23}$ e Faaborg et $\mathrm{al}^{24}$, estímulos nociceptivos abaixo do nível de lesão como uma infecção urinária ou até roupas e sapatos apertados podem induzir a uma crise de DA.

Estudos $^{9,12}$ descrevem que estes estímulos desencadeiam a hiperestimulação do SNAS, ocorrendo liberação de noradrenalina e dopamina que geram uma vasoconstrição, como consequência ocorre a elevação da PA. Como forma de tentativa para diminuir os níveis pressóricos, a uma ativação dos centros dos baroreceptores aórticos e carotídeos e dos centros vasomotores localizado no tronco encefálico, para um estímulo inibitório.

Nesse sentido, a DA pode ser desencadeada por reflexos medulares que se mantêm intactos apesar da lesão presente, e nesse contexto, um estímulo nocivo abaixo do nível da lesão ocasionariam impulsos aferentes que levam a uma resposta simpática generalizada e excessiva. Em seguida, é iniciada uma resposta adrenérgica, onde será realizada a ativação dos baroreceptores que se localizam nos seios carotídeos, acima do 
nível da lesão que irão desencadear uma reação do SNAP causando uma ação vasodilatadora para gerar a diminuição da frequência cardíaca ${ }^{12}$.

Vale mencionar que alguns pacientes de DA não sabiam a respeito de sua condição clínica, apesar de referir seus sinais e sintomas, mostrando a importância de ter melhorias na educação nesses indivíduos sobre sua patologia com objetivo de identificar sua sintomatologia e como saber procurar assistência para sua condição ${ }^{15}$.

No estudo realizado por Fougere et $\mathrm{al}^{25}$, com 22 indivíduos homens e mulheres com idade entre 18 e 65 anos com > 1 ano pós-lesão traumático em nível de T6. Foram submetidos a procedimento de OnabotulinumtoxinA, no qual observou que esse meio de tratamento na bexiga afetou de forma positiva os eventos de DA, no qual viu uma diminuição na gravidade dessa patologia por redução da PAS máxima quanto da PASD e a frequência de eventos relacionados à bexiga.

De forma resolutiva é preciso seguir um conjunto de atitudes e intervenções que devem ser realizadas pela ordem: 1 . sentar o paciente; 2 . fiscalizar os sinais vitais; 3. reconhecer e excluir o estímulo nociceptivo, que nesse caso, pode ser remover a roupa apertada ou caso o utente esteja com retenção urinária deve ser feito o procedimento de esvaziamento ou impactação fecal. Se não obtiver melhoras do quadro clínico, devese investigar a causa menos frequentes de DA, e, se a PAS estiver muito elevada, o paciente deve ser medicado com anti-hipertensos de ação ligeira e curta duração. Recomenda-se que mesmo diante da reversão do quadro, deve-se monitorar o paciente durante 2 horas. Na grande maioria dos casos é facilmente revertido ${ }^{9-18}$.

\section{CONCLUSÃO}

Foi possível observar que a DA é uma síndrome de grande fatalidade e de imensa importância clínica, no qual os autores mostram que suas sequelas limitam os afetados de forma bastante notória. Essa patologia necessita de grande assistência dos profissionais de saúde inclusive da fisioterapia, que tem uma atuação pouco conhecida perante os mesmos, porém de grande importância e eficácia na melhora dos sinais e sintomas.

É notório que esses pacientes apresentam uma constelação de sintomatologia, no qual vários autores corroboram em seus estudos, por isso faz necessário um cuidado especializado e adequado nesses indivíduos. 
Vale mencionar alguns pontos de importância desses pacientes, como a educação para que os mesmo possa se ajudar em seu processo saúde e doença, o uso da toxina botulínica, que além de minimizar a espasticidade também auxilia os profissionais de saúde em suas condutas, e não menos importante às intervenções do trato urinário que segundo os estudos encontrados são um dos maiores causadores da DA.

Contudo, observou-se que há uma grande escassez de estudos referente o tema, e que se fazem necessárias mais pesquisas para disseminar mais essa desordem e fazer com que seja mais conhecida perante os profissionais de saúde com objetivo de aperfeiçoar os cuidados aos portadores de DA.

\section{REFERENCIAS}

1. Brandão $\mathrm{CH}$. Efeitos da estimulação magnética transcraniana de alta frequência sobre a função motora na lesão medular incompleta: Estudos de casos [monografia]. Campina Grande: Universidade Estadual da Paraíba; 2017. 33 p.

2. Magalhães SR, Carvalho ZM, Andrade LM, Pinheiro AK, Studart RM. Influência da espiritualidade, religião e crenças na qualidade de vida de pessoas com lesão medular. Texto \& contexto enferm. 2015;24(3):792-800.

3. Nardone R, Orioli A, Golaszewski S, Brigo F, Sebastianelli L, Holler Y. Passive cycling in neurorehabilitation after spinal cord injury: A review. J Spinal Cord Med. 2017; 40(1):8-16.

4. Santos EA, Alves VL, Ramos S, Frangella VS. Análise do hábito alimentar e do estado nutricional de pacientes com lesão medular após intervenção nutricional. Acta Fisiatr. 2014; 21(3):121-31.

5. Thomas FP, Araújo DF. Manuseio da disreflexia autonômica em pacientes com lesões medulares. Lesões medulares clínicas e experimentais. Imprensa Universitária da Universidade Federal do Ceará (UFC); 2016. 306 p.

6. Damiani D. Choque neurogênico: manejo clínico e suas particularidades na sala de emergência. Arq bras neurocir. 2016. 10 p.

7. França IS, Coura AS, Sousa FS, Almeida PC, Pagliuca LM. Qualidade de vida em pacientes com lesão medular. Rev Gaúcha Enferm. 2013; 34(1):155-63.

8. Chan M. International Perspectives on Spinal Cord Injury. Geneva: World Health Organization; 2013. $250 \mathrm{p}$. 
9. Roque V, Cunha I, Rocha A, Andrade MJ. Disfunções Autonómicas após Lesão Medular. Portuguese Journal of Physical and Rehabilitation Medicine. 2013; 24(2)1-9.

10. Allen K J, Leslie SW. Autonomic Dysreflexia: StatPearls [Internet]. 2018 Jul-Jan [cited Jul 2018] Available from: https: //www.ncbi.nlm.nih.gov/books/NBK482434/.

11. Strcić N, Markic D. The knowledge about autonomic dysreflexia among nursing and physiotherapy students. J Spinal Cord Med. 2018.10 p.

12. Lagarto F, Pina P. Disreflexia Autonômica no Peri-operatório. Rev Soc Port Anestesiol. 2016; 25(2):69-72.

13. Wang K, Duan S, Wen X, Wang W, Fang S, Qi D. Angiotensin II system in the nucleus tractus solitarii contributes to autonomic dysreflexia in rats with spinal cord injury. PLoS one. 2017; 12(7).

14. Khanna K, Theologis AA, Tay B. Autonomic dysreflexia caused by cervical stenosis. Spinal Cord Series and Cases. 2017;(3).

15. Inskip JA, Lucci VM, McGath MS, Willms R, Claydon VE. A Community Perspective on Bowel Management and Quality of Life after Spinal Cord Injury: The Influence of Autonomic Dysreflexia. J Neurotrauma. 2018; 35(9):1091-105.

16. Andrade LT, Araújo EG, Andrade KR, Souza DR, Garcia TR, Chianca TC. Disreflexia autonômica e intervenções de enfermagem para pacientes com lesão medular. Rev Esc Enferm USP. 2013; (1):93-100.

17. Fabro AS, Mejia M, Nemunaitis G. An investigation of the relationship between autonomic dysreflexia and intrathecal baclofen in patients with spinal cord injury. $J$ Spinal Cord Med. 2017;41(1):102-105.

18. Ministério da Saúde (BR), Secretaria de Atenção à Saúde, Política Nacional de Humanização da Atenção e Gestão do SUS. Departamento de Ações Programáticas Estratégicas e Departamento de Atenção Especializada. Diretrizes de Atenção à pessoa com lesão medular. Brasília (DF): Ministério da Saúde; 2013.

19. Lee ES, Joo AM. Prevalence of Autonomic Dysreflexia in Patients with Spinal Cord Injury above T6. BioMed Research International. 2017.6 p.

20. Solinsky R, Kirshblum SC, Burns S. Exploring detailed characteristics of autonomic dysreflexia. J Spinal Cord Med. 2017; 41(5):549-555. 
21. Eldahan KC, Rabchevsky AG. Autonomic dysreflexia after spinal cord injury: Systemic pathophysiology and methods of management. Auton Neurosci. 2018; 209:59-70.

22. Vaidyanathan S, Soni BM, Oo T, Hughes PL, Singh G. Missed signs of autonomic dysreflexia in a tetraplegic patient after incorrect placement of urethral Foley catheter: a case report. Patient Saf Surg. 2014; 22:8-44.

23. Huang $\mathrm{YH}$. Blood pressure and age associated with silent autonomic dysreflexia during urodynamicexaminations in patients with spinal cord injury. Spinal Cord. 2013; 51(5):401-5.

24. Faaborg PM. Autonomic dysreflexia during bowel evacuation procedures and bladder filling in subjects with spinal cord injury. Spinal Cord. 2014; 52(6):494-8.

25. Fougere RJ, Currie KD, Nigro MK, Stothers L, Rapoport D, Krassioukov AV. Reduction in Bladder Related Autonomic Dysreflexia after OnabotulinumtoxinA Treatment in Spinal Cord Injury. J Neurotrauma. 2016; 33(18):1651-7.

ANEXO A - Lista de artigos utilizados na revisão integrativa.

\begin{tabular}{|c|c|c|c|c|}
\hline AUTORES & TÍTULO & AMOSTRA & OBJETIVO & RESULTADOS \\
\hline $\begin{array}{l}\text { Strčić N, } \\
\text { Markić D11 }\end{array}$ & $\begin{array}{l}\text { The knowledge } \\
\text { about autonomic } \\
\text { dysreflexia among } \\
\text { nursing and } \\
\text { physiotherapy } \\
\text { students }\end{array}$ & $\begin{array}{c}\text { Estudo de } \\
\text { questionário de } \\
\text { centro único, que } \\
\text { continha } 43 \\
\text { estudantes de } \\
\text { Enfermagem e } 48 \text { de } \\
\text { fisioterapia. }\end{array}$ & $\begin{array}{l}\text { Detectar o nível de } \\
\text { conhecimento da } \\
\text { DA. }\end{array}$ & $\begin{array}{l}\text { O conhecimento da } \\
\text { DA foi julgado como } \\
\text { ruim ou nenhum em } \\
73,6 \% \text { dos alunos. }\end{array}$ \\
\hline $\begin{array}{l}\text { Lee E S, Joo } \\
\text { A M C } \text { C }^{19}\end{array}$ & $\begin{array}{c}\text { Prevalence of } \\
\text { Autonomic } \\
\text { Dysreflexia in } \\
\text { Patients with } \\
\text { Spinal } \\
\text { Cord Injury above } \\
\text { T6 }\end{array}$ & $\begin{array}{c}\text { Estudo Transversal } \\
\text { de prevalência, } 28 \\
\text { pacientes } \\
\text { diagnosticados com } \\
\text { LM acima de T6 } \\
\text { foram inscritos. }\end{array}$ & $\begin{array}{c}\text { Investigar a } \\
\text { prevalência da DA } \\
\text { em pacientes com } \\
\text { LM acima de T6. }\end{array}$ & $\begin{array}{l}\text { A DA ocorreu em } 26 \\
\text { pacientes e os } \\
\text { eventos aconteceram } \\
5,8 \pm 4,7 \text { vezes. } \\
\text { Achados sugerem a } \\
\text { perda da PA noturna } \\
\text { em pacientes com } L M \text {. } \\
\text { Os resultados do } \\
\text { questionário de } \\
\text { disfunção autonômica } \\
\text { após } L M \text { revelaram } \\
\text { que } 16 \text { dos pacientes } \\
\text { avaliados eram } \\
\text { sintomáticos, } \\
\text { enquanto } 12 \text { eram } \\
\text { assintomáticos. }\end{array}$ \\
\hline $\begin{array}{l}\text { Roque V et } \\
\text { al }^{9}\end{array}$ & $\begin{array}{c}\text { Disfunções } \\
\text { Autonômicas após } \\
\text { Lesão Medular }\end{array}$ & $\begin{array}{l}\text { Trata-se de um } \\
\text { artigo de revisão } \\
\text { literária das } \\
\text { disfunções } \\
\text { autonômicas e do } \\
\text { seu tratamento, }\end{array}$ & $\begin{array}{c}\text { Revisar as } \\
\text { disfunções } \\
\text { autonômicas, } \\
\text { tratamento, assim } \\
\text { como das múltiplas } \\
\text { complicações que }\end{array}$ & $\begin{array}{l}\text { As LM acarretam } \\
\text { graves complicações, } \\
\text { não só motoras e } \\
\text { sensitivas, mas } \\
\text { também autonômicas } \\
\text { com risco de vida }\end{array}$ \\
\hline
\end{tabular}




\begin{tabular}{|c|c|c|c|c|}
\hline & & $\begin{array}{l}\text { onde não foi } \\
\text { identificada a } \\
\text { amostra. }\end{array}$ & $\begin{array}{l}\text { acarretam no } \\
\text { processo de } \\
\text { reabilitação. }\end{array}$ & inerente. \\
\hline $\begin{array}{l}\text { Lagarto F, } \\
\text { Pina } \mathrm{P}^{12}\end{array}$ & $\begin{array}{c}\text { Disreflexia } \\
\text { Autonómica no } \\
\text { Peri-operatório }\end{array}$ & $\begin{array}{l}\text { Uma doente de } 48 \\
\text { anos, com lesão } \\
\text { traumática } \\
\text { vertebro-medular, } \\
\text { ao nível da } \\
\text { transição cervico- } \\
\text { torácica, submetida } \\
\text { a injeção intra- } \\
\text { vesical de toxina } \\
\text { botulínica, sob } \\
\text { anestesia geral } \\
\text { inalatória. }\end{array}$ & $\begin{array}{c}\text { Reforçar a } \\
\text { importância das } \\
\text { medidas } \\
\text { preventivas, } \\
\text { nomeadamente a } \\
\text { preferência pela } \\
\text { anestesia regional } \\
\text { em cirurgia } \\
\text { urológica nos } \\
\text { doentes com lesão } \\
\text { vertebro-medular. }\end{array}$ & $\begin{array}{l}\text { No intra-operatório } \\
\text { foi observado sinais e } \\
\text { sintomas compatíveis } \\
\text { com episódio de DA. } \\
\text { Apesar da rápida } \\
\text { identificação e } \\
\text { suspensão dos fatores } \\
\text { desencadeantes, o } \\
\text { quadro clínico } \\
\text { reverteu apenas no } \\
\text { pós-operatório, depois } \\
\text { da administração de } \\
\text { labetalol. }\end{array}$ \\
\hline $\begin{array}{l}\text { Wang K et } \\
\mathrm{al}^{13}\end{array}$ & $\begin{array}{l}\text { Angiotensin II } \\
\text { system in the } \\
\text { nucleus tractus } \\
\text { solitarii } \\
\text { contributes to } \\
\text { autonomic } \\
\text { dysreflexia in rats } \\
\text { with spinal cord } \\
\text { injury }\end{array}$ & $\begin{array}{l}\text { Estudo de caso de } \\
40 \text { ratos machos }\end{array}$ & $\begin{array}{l}\text { Determinar o papel } \\
\text { da regulação } \\
\text { barorreflexa } \\
\text { prejudicada pelo } \\
\text { núcleo do trato } \\
\text { solitário na } \\
\text { ocorrência de DA } \\
\text { em um modelo de } \\
\text { rato. }\end{array}$ & $\begin{array}{l}\text { A ativação do sistema } \\
\text { Ang II no NTS pode } \\
\text { prejudicar a pressão } \\
\text { arterial barorreflexa e } \\
\text { contribuir para a DA } \\
\text { após a LM. }\end{array}$ \\
\hline $\begin{array}{l}\text { Khanna K, } \\
\text { Theologis } \\
\text { AA, Tay B } B^{14}\end{array}$ & $\begin{array}{c}\text { Autonomic } \\
\text { dysreflexia caused } \\
\text { by cervical } \\
\text { stenosis }\end{array}$ & $\begin{array}{l}\text { Relato de caso de } \\
\text { um homem de } 73 \\
\text { anos de idade com } \\
\text { estenose cervical. }\end{array}$ & $\begin{array}{l}\text { Alertar os clínicos } \\
\text { para uma potencial } \\
\text { associação entre } \\
\text { DA e estenose } \\
\text { cervical, que pode } \\
\text { existir fora do } \\
\text { domínio do LM. }\end{array}$ & $\begin{array}{l}\text { A DA pode ser causada } \\
\text { por estenose cervical, } \\
\text { na qual a compressão } \\
\text { cervical causou uma } \\
\text { ruptura no controle } \\
\text { regulatório do sistema } \\
\text { simpático, neurônios } \\
\text { pré-ganglionares, } \\
\text { resultando em } \\
\text { sintomas } \\
\text { autonômicos. }\end{array}$ \\
\hline $\begin{array}{l}\text { Inskip J } \mathrm{J} \\
\mathrm{al}^{15}\end{array}$ & $\begin{array}{c}\text { A Community } \\
\text { Perspective on } \\
\text { Bowel Management } \\
\text { and Quality of Life } \\
\text { after Spinal Cord } \\
\text { Injury: } \\
\text { The Influence of } \\
\text { Autonomic } \\
\text { Dysreflexia }\end{array}$ & $\begin{array}{l}\text { Foi realizada uma } \\
\text { pesquisa da } \\
\text { comunidade online } \\
\text { de indivíduos com } \\
\text { lesão medular. } \\
\text { Aqueles com lesão } \\
\text { igual ou superior } \\
\text { T7 foram } \\
\text { considerados em } \\
\text { risco para DA. } \\
\text { Foram recebidas } \\
\text { respostas de } 287 \\
\text { indivíduos com LM. }\end{array}$ & $\begin{array}{c}\text { Descrever as } \\
\text { relações entre } \\
\text { cuidados } \\
\text { intestinais, DA e } \\
\text { qualidade de vida } \\
\text { em indivíduos com } \\
\text { LM. }\end{array}$ & $\begin{array}{l}\text { A DA interferiu nas } \\
\text { atividades da vida } \\
\text { diária em } 51 \% \text {. As } \\
\text { durações de cuidados } \\
\text { intestinais e DA mais } \\
\text { grave foram } \\
\text { associadas com menor } \\
\text { qualidade de vida. }\end{array}$ \\
\hline $\begin{array}{l}\text { Andrade } \mathrm{LT} \text {, } \\
\text { et } \mathrm{al}^{16}\end{array}$ & $\begin{array}{c}\text { Disreflexia } \\
\text { autonômica e } \\
\text { intervenções de } \\
\text { enfermagem para } \\
\text { pacientes com } \\
\text { lesão medular }\end{array}$ & $\begin{array}{l}465 \text { prontuários de } \\
\text { pacientes } \\
\text { com LM em processo } \\
\text { de reabilitação }\end{array}$ & $\begin{array}{c}\text { Identificar } \\
\text { diagnósticos e } \\
\text { intervenções } \\
\text { de enfermagem } \\
\text { para o cuidado de } \\
\text { pacientes } \\
\text { com LM. }\end{array}$ & $\begin{array}{l}\text { Neste estudo, ficou } \\
\text { evidente que retirar o } \\
\text { estímulo causador foi } \\
\text { à terapêutica mais } \\
\text { eficaz. }\end{array}$ \\
\hline $\begin{array}{l}\text { Fabro AS, } \\
\text { Mejia M, }\end{array}$ & $\begin{array}{l}\text { An investigation of } \\
\text { the relationship }\end{array}$ & $\begin{array}{l}\text { Revisões de gráfico } \\
\text { retrospectivo, que }\end{array}$ & $\begin{array}{l}\text { Estudar a relação } \\
\text { entre disreflexia }\end{array}$ & $\begin{array}{c}\text { Dos } 34 \text { indivíduos, } 25 \\
\text { apresentaram DA }\end{array}$ \\
\hline
\end{tabular}




\begin{tabular}{|c|c|c|c|c|}
\hline$\underset{\mathrm{G}^{17}}{\text { Nemunaitis, }}$ & $\begin{array}{c}\text { between } \\
\text { autonomic } \\
\text { dysreflexia and } \\
\text { intrathecal } \\
\text { baclofen in } \\
\text { patients with } \\
\text { spinal cord injury }\end{array}$ & $\begin{array}{l}\text { possuiu } 34 \text { sujeitos, } \\
\text { foram revisadas os } \\
\text { registros médicos } \\
\text { dos pacientes antes } \\
\text { e após os } \\
\text { procedimentos e foi } \\
\text { registrado os fatores } \\
\text { de risco para a DA. }\end{array}$ & $\begin{array}{l}\text { autonômica e } \\
\text { baclofeno } \\
\text { intratecal em } \\
\text { pacientes com } \\
\text { lesão da medula } \\
\text { espinal. }\end{array}$ & $\begin{array}{c}\text { antes da colocação da } \\
\text { bomba de ITB e } \\
\text { apenas } 2 \text { após.Foi } \\
\text { mostrada uma } \\
\text { redução significativa } \\
\text { dos episódios } \\
\text { sintomáticos de DA } \\
\text { após LM, mesmo } \\
\text { naqueles com fatores } \\
\text { de risco adicionais } \\
\text { para o } \\
\text { desenvolvimento de } \\
\text { DA. }\end{array}$ \\
\hline $\begin{array}{l}\text { Solinsky R, } \\
\text { Kirshblum } \\
\text { SC, Burns } \text { S }^{20}\end{array}$ & $\begin{array}{l}\text { Exploring detailed } \\
\text { characteristics of } \\
\text { autonomic } \\
\text { dysreflexia }\end{array}$ & $\begin{array}{c}\text { Análise do conjunto } \\
\text { de dados coletados } \\
\text { prospectivamente } \\
\text { da DA. Os } \\
\text { participantes foram } \\
78 \text { pacientes do } \\
\text { sexo masculino com } \\
\text { LM que } \\
\text { experimentaram DA. }\end{array}$ & $\begin{array}{c}\text { Identificar a clínica } \\
\text { e fisiopatologia da } \\
\text { DA em pacientes } \\
\text { com } \\
\text { LM. }\end{array}$ & $\begin{array}{l}\text { Foram identificados } \\
445 \text { episódios de DA, } \\
\text { no qual comumente se } \\
\text { apresentava a } \\
\text { taquicardia como } \\
\text { sinal. O estudo } \\
\text { sugeriu mais } \\
\text { investigações sobre os } \\
\text { efeitos da cronicidade } \\
\text { da LM e do manejo } \\
\text { farmacológico. }\end{array}$ \\
\hline $\begin{array}{c}\text { Eldahan KC, } \\
\text { Rabchevsky } \\
\text { AG }^{21}\end{array}$ & $\begin{array}{c}\text { Autonomic } \\
\text { dysreflexia after } \\
\text { spinal cord injury: } \\
\text { Systemic } \\
\text { pathophysiology } \\
\text { and methods of } \\
\text { management }\end{array}$ & $\begin{array}{c}\text { Avenidas de } \\
\text { pesquisa } \\
\text { contemporânea são } \\
\text { apresentadas para } \\
\text { entender melhor as } \\
\text { contribuições } \\
\text { relativas dos } \\
\text { mecanismos } \\
\text { subjacentes }\end{array}$ & $\begin{array}{c}\text { Revisar os } \\
\text { mecanismos } \\
\text { fisiopatológicos } \\
\text { pertinentes ao } \\
\text { desenvolvimento da } \\
\text { DA, discutir os } \\
\text { efeitos sistêmicos } \\
\text { da DA recorrente e } \\
\text { tratamentos } \\
\text { farmacológicos. }\end{array}$ & $\begin{array}{c}\text { A DA pode ter um } \\
\text { impacto significativo } \\
\text { na qualidade de vida } \\
\text { diária e, se não } \\
\text { tratada de forma } \\
\text { adequada e oportuna, } \\
\text { essa síndrome } \\
\text { hipertensiva pode ter } \\
\text { consequências } \\
\text { cardiofisiológicas e } \\
\text { sistêmicas deletérias. }\end{array}$ \\
\hline $\begin{array}{l}\text { Vaidyanathn } \\
\text { S et } \mathrm{al}^{22}\end{array}$ & $\begin{array}{c}\text { Missed signs of } \\
\text { autonomic } \\
\text { dysreflexia in a } \\
\text { tetraplegic patient } \\
\text { after incorrect } \\
\text { placement of } \\
\text { urethral Foley } \\
\text { catheter: a case } \\
\text { report }\end{array}$ & $\begin{array}{l}\text { Um paciente com } \\
\text { lesão medular, que } \\
\text { desenvolveu DA } \\
\text { após a colocação } \\
\text { incorreta do cateter } \\
\text { de Foley uretral. }\end{array}$ & $\begin{array}{l}\text { Apresentar o caso } \\
\text { do paciente com } \\
\text { LM que } \\
\text { desenvolveu DA } \\
\text { após a colocação } \\
\text { incorreta do } \\
\text { cateter de Foley } \\
\text { uretral. Mostrar os } \\
\text { sinais e sintomas da } \\
\text { DA, bem como sua } \\
\text { significância } \\
\text { paciente } \\
\text { tetraplégico. }\end{array}$ & $\begin{array}{l}\text { A inserção de um } \\
\text { cateter em paciente } \\
\text { tetraplégico deve ser } \\
\text { realizada por um } \\
\text { profissional de saúde } \\
\text { que está familiarizado } \\
\text { com o espasmo do } \\
\text { colo da bexiga. } \\
\text { Instalações para } \\
\text { tomografia } \\
\text { computadorizada } \\
\text { urgente devem estar } \\
\text { disponíveis para } \\
\text { verificar a posição do } \\
\text { cateter de Foley em } \\
\text { pacientes com LM } \\
\text { quando se manifesta } \\
\text { sinais e sintomas de } \\
\text { DA após a inserção de } \\
\text { um cateter uretral. }\end{array}$ \\
\hline Huang $\mathrm{Y} \mathrm{H}^{23}$ & $\begin{array}{l}\text { Blood pressure and } \\
\text { age associated } \\
\text { with silent }\end{array}$ & $\begin{array}{c}\text { Um estudo } \\
\text { observacional que } \\
\text { continha um total }\end{array}$ & $\begin{array}{c}\text { Investigar os } \\
\text { fatores associados } \\
\text { à DA silenciosa }\end{array}$ & $\begin{array}{c}\text { Pacientes } \\
\text { sintomáticos com DA } \\
\text { tenderam a ter }\end{array}$ \\
\hline
\end{tabular}




\begin{tabular}{|c|c|c|c|c|}
\hline & $\begin{array}{c}\text { autonomic } \\
\text { dysreflexia during } \\
\text { urodynamic } \\
\text { examinations in } \\
\text { patients with } \\
\text { spinal cord injury }\end{array}$ & $\begin{array}{l}\text { de } 42 \text { pacientes, } 21 \\
\text { nos grupos } \\
\text { sintomáticos e } 21 \text { no } \\
\text { silencioso, foi } \\
\text { analisado. }\end{array}$ & $\begin{array}{c}\text { durante a } \\
\text { urodinâmica na LM. }\end{array}$ & $\begin{array}{l}\text { elevação significativa } \\
\text { da PA diastólica e } \\
\text { incrementos mais } \\
\text { rápidos da PA sistólica } \\
\text { / PA diastólica. O } \\
\text { envelhecimento } \\
\text { diminui os sintomas } \\
\text { da DA e a elevação da } \\
\text { PA diastólica }\end{array}$ \\
\hline Faaborg PM ${ }^{24}$ & $\begin{array}{c}\text { Autonomic } \\
\text { dysreflexia during } \\
\text { bowel evacuation } \\
\text { procedures } \\
\text { and bladder filling } \\
\text { in subjects with } \\
\text { spinal cord injury }\end{array}$ & $\begin{array}{l}\text { Ensaio clínico } \\
\text { randomizado } \\
\text { controlado, que } \\
\text { constituiu de } 8 \\
\text { indivíduos com LM } \\
\text { alta igual ou } \\
\text { superior a T6 e } \\
\text { história prévia de } \\
\text { DA foram } \\
\text { comparados com } 3 \\
\text { sujeitos com LM } \\
\text { entre T10 e L2. }\end{array}$ & $\begin{array}{l}\text { Investigar respostas } \\
\text { autonômicas à } \\
\text { evacuação retal } \\
\text { digital, irrigação } \\
\text { transanal com } \\
500 \mathrm{ml} \text { e } \\
\text { cistometria de } \\
\text { enchimento em LM. }\end{array}$ & $\begin{array}{c}\text { O controle do } \\
\text { intestino e da bexiga } \\
\text { causou DA em LM } \\
\text { alta. A resposta é } \\
\text { menos grave durante } \\
\text { a irrigação transanal } \\
\text { do que durante a } \\
\text { cistometria de } \\
\text { enchimento ou } \\
\text { evacuação retal } \\
\text { digital. }\end{array}$ \\
\hline $\begin{array}{l}\text { Fougere RJ } \\
\text { et } \mathrm{al}^{10}\end{array}$ & $\begin{array}{c}\text { Reduction in } \\
\text { Bladder-Related } \\
\text { Autonomic } \\
\text { Dysreflexia after } \\
\text { Onabotulinumtoxin } \\
\text { aA Treatment in } \\
\text { Spinal Cord Injury }\end{array}$ & $\begin{array}{c}\text { Este estudo usou um } \\
\text { design prospectivo e } \\
\text { aberto de } 2013 \text { para } \\
2014,12 \text { homens e } 5 \\
\text { mulheres com LM } \\
\text { traumática crônica } \\
\text { no } 6^{\circ} \text { nível torácico } \\
\text { ou acima dele e DA } \\
\text { concomitante e } \\
\text { hiperatividade } \\
\text { neurogênica do } \\
\text { detrusor. }\end{array}$ & $\begin{array}{c}\text { Avaliar } \\
\text { quantitativamente } \\
\text { a eficácia de um } \\
\text { ciclo de injeção de } \\
\text { onabotulinumtoxina } \\
\text { A. A gravidade e a } \\
\text { frequência de } \\
\text { eventos de DA } \\
\text { relacionados à } \\
\text { bexiga e melhoria } \\
\text { da qualidade de } \\
\text { vida. }\end{array}$ & $\begin{array}{c}\text { Foi demonstrado uma } \\
\text { redução na gravidade } \\
\text { e frequência globais } \\
\text { de eventos } \\
\text { relacionados com a } \\
\text { bexiga, } 1 \text { mês após } \\
\text { isso do Botox para } \\
\text { hiperatividade } \\
\text { neurogênica do } \\
\text { detrusor. O } \\
\text { tratamento é viável } \\
\text { para o alívio bem- } \\
\text { sucedido de episódios } \\
\text { de DA e, melhoria dos } \\
\text { riscos para a saúde } \\
\text { relacionada com o } \\
\text { com a LM. }\end{array}$ \\
\hline
\end{tabular}

Conflito de interesses: Os autores declaram não haver conflito de interesses.

\section{Participação dos autores:}

- Concepção: Soares LMMM.

- Desenvolvimento: Vasconcelos LA, Souza LKA, Moura RF, Freire LPV.

- Redação e revisão: Vasconcelos LA, Souza LKA, Moura RF, Soares LMMM.

Como citar este artigo: Vasconcelos LA, Souza LKA, Moura RF, Freire LPV, Soares LMMM. Conceitos, manifestações clínicas e cuidados imediatos na disreflexia autonômica. Journal Health NPEPS. 2018 jul-dez; 3(2): 618633.

Submissão: $28 / 07 / 2018$

Aceito: $01 / 11 / 2018$

Publicado: 30/12/2018 